\title{
Melatonin alters the secondary metabolite profile of grape berry skin by promoting VvMYB14-mediated ethylene biosynthesis
}

\author{
Wanyun Ma', Lili Xu', Shiwei Gao', Xingning Lyu', Xiaolei Cao ${ }^{1}$ and Yuxin Yao (i)
}

\begin{abstract}
The interplay between melatonin and ethylene in the regulation of fruit metabolism and the underlying molecular mechanism of this interplay remain largely unclear. Here, widely targeted metabolomics analysis revealed a total of 464 metabolites present in berry skin. Among them, 27 significantly differentially accumulated metabolites (DAMs) were produced in response to melatonin treatment in the presence or absence of 1-MCP. Most of the DAMs were secondary metabolites, including flavonoids, phenolic acids, stilbenes, and flavonols. Additionally, the accumulation of 25 DAMs was regulated by melatonin via ethylene. RNA-seq analysis indicated that melatonin primarily regulated the pathways of plant hormone signal transduction and secondary metabolite biosynthesis via ethylene. Gene-metabolite association analysis showed that melatonin regulated the expression of the VVSTS1, VVF3H, VVLAR2, and VVDFR genes, suggesting that these genes may play key roles in regulating secondary metabolites in the skin; additionally, VVMYB14 and VVACS1 were suggested to be involved in the regulation of secondary metabolites. Further experiments revealed that melatonin induced the expression of VVMYB14 and that VVMYB14 increased ethylene production by transcriptionally activating VVACS1, thereby affecting the accumulation of secondary metabolites. Collectively, melatonin promotes ethylene biosynthesis and alters secondary metabolite accumulation through the regulation of VVACS1 by VVMYB14.
\end{abstract}

\section{Introduction}

Grapevine is one of the most important fruit crop species worldwide, and the quality of grape berries mainly depends on their primary and secondary metabolites; moreover, the chemical composition of berries is primarily influenced by secondary metabolites, including alkaloids, terpenes, phenolics, and volatiles ${ }^{1}$. Grape berries are a rich source of natural antioxidant compounds (mainly polyphenols) that contribute to more than half of the global polyphenol market ${ }^{2}$. Grape berries are nonclimacteric fruits, and several signaling molecules, including melatonin and ethylene, participate in berry

\footnotetext{
Correspondence: Yuxin Yao (yaoyx@sdau.edu.cn)

${ }^{1}$ State Key Laboratory of Crop Biology, Collaborative Innovation Center of Fruit \& Vegetable Quality and Efficient Production, College of Horticulture Science and Engineering, Shandong Agricultural University, Tai-An, Shandong 271018, China
}

metabolism and ripening regulation $^{3}$, although the mechanism underlying the ripening of nonclimacteric fruits, including grape berries, remains largely unclear.

The ethylene-mediated regulatory network of climacteric fruits is well known. In contrast, only a few studies have shown that nonclimacteric fruits, including those of grape, strawberry, and cherry, have a fully functional ethylene biosynthesis pathway. Peak ethylene production occurs during early development of grape berries and strawberry fruits; ${ }^{4,5}$ this pattern is typical for nonclimacteric fruits. Metabolomic analysis has shown that ethylene regulates a wide range of metabolites in nonclimacteric Capsicum fruits ${ }^{6}$. Additionally, the discovery of 1-methylcyclopropene (1-MCP) as a specific inhibitor of ethylene action has provided a powerful tool for elucidating ripening and senescence mechanisms in climacteric and nonclimacteric fruits and vegetables ${ }^{7,8}$. For 
example, both exogenous ethylene and 1-MCP alter ripening-related parameters, including anthocyanin accumulation, in grape ${ }^{9}$. Moreover, exogenous ethylene and 1-MCP promote and inhibit, respectively, the postharvest ripening of sweet cherry fruits ${ }^{10}$. Studies of nonclimacteric fruits challenged with 1-MCP have identified both ethylene-dependent and ethylene-independent ripening processes ${ }^{7}$.

Melatonin (N-acetyl-5-methoxytryptamine, MT) is a low-molecular-weight indole amine synthesized from Ltryptophan and functions as a pleiotropic molecule with diverse functions in plants ${ }^{11}$. It has been revealed that MT plays a role in regulating fruit ripening or postharvest senescence across various plant systems, such as those of grape, tomato, and banana ${ }^{12-14}$. In addition, MT has been reported to increase the content of phenols, anthocyanins, and flavonoids in grape berries ${ }^{13}$. Postharvest treatment with MT increases total phenols and anthocyanins in strawberry fruits ${ }^{15}$ and delays the loss of total anthocyanins, flavonoids, and phenols in litchi fruits ${ }^{16}$. Furthermore, MT increases the content of soluble sugars, particularly sucrose and sorbitol, in pear fruits ${ }^{17}$. Therefore, MT promotes fruit ripening and alters metabolite accumulation; however, the mechanism underlying these actions remains largely unknown. Several studies have shown that MT may function through interactions with other hormones, including ABA, ethylene, auxin, and cytokinins $^{18}$. Our previous study indicated that MT treatment promotes grape berry ripening in part via other signaling molecules, such as $\mathrm{ABA}, \mathrm{H}_{2} \mathrm{O}_{2}$ and, in particular, ethylene; ${ }^{5}$ additionally, MT increases the polyphenol content of berries via ethylene signaling ${ }^{13}$.

Additionally, several studies have demonstrated that MYB14 is involved in the regulation of metabolites. VvMYB14 is reported to transcriptionally regulate stilbene biosynthesis by specifically activating the promoters of the STS gene in grapevine ${ }^{19}$. Analysis of overexpression and mutant plants showed that MtMYB14 is related to proanthocyanidin accumulation in the hairy roots and seeds of Medicago truncatula ${ }^{20}$. Moreover, ZmMYB14 functions as a key regulator of ZmBT1 and is closely related to the biosynthesis of starch by transcriptionally activating the expression of six starch synthesis-related genes in maize ${ }^{21}$. Although some target genes of MYB14 have been identified, the mechanism underlying the broad role of MYB14 in regulating metabolites needs further investigation. Additionally, in grape, $V v M Y B 14$ was found to be significantly induced by MT in our previous study ${ }^{13}$, but it remains unclear whether VvMYB14 regulates secondary metabolism in response to MT.

To date, little is known about the global changes in metabolites caused by MT and about the interplay of MT and ethylene, particularly the underlying molecular mechanism. Therefore, the objective of the present study was to determine whether MT promotes ethylene biosynthesis and alters secondary metabolite accumulation through the regulation of VvACS1 by VvMYB14. 1-MCP was applied to inhibit ethylene signaling in control and MT-treated berry skins. Widely targeted metabolomics and RNA-seq analysis were used to provide a comprehensive identification of metabolites and genes induced in response to melatonin in the presence or absence of 1MCP. Transcriptional activation assays of $V v A C S 1$ by VvMYB14 and overexpression and suppression of $V v M Y B 14$ in grape calli were performed to demonstrate the role of $V v M Y B 14$ in mediating the interaction of melatonin and ethylene.

\section{Results}

MT treatment of preveraison grape berries promotes ethylene production in the skin, which is not affected by 1MCP

TSS, titratable acid, and anthocyanin contents were measured to evaluate the occurrence of veraison (onset of berry ripening). Anthocyanins and TSS began to accumulate, and titratable acid began to decrease at 80 days after blooming (DAB) (Fig. 1A), indicating the occurrence of veraison at approximately this time point. The peaks of $\mathrm{MT}$ and ethylene production were detected at $70 \mathrm{DAB}$ in the control berry skins. Afterward, the MT content and ethylene production rate declined sharply, and MT was undetectable past $120 \mathrm{DAB}$ (Fig. 1B, C). MT treatment at 70 DAB largely increased MT levels in the berry skin and led to an 8.59-fold increase at $80 \mathrm{DAB}$ compared with the level in the control skin (Fig. 1B). Additionally, MT and, in particular, ethephon, significantly increased ethylene production from 80 to $110 \mathrm{DAB}$ and caused $47.12 \%$ and $194.99 \%$ increases in ethylene production, respectively, at 80 DAB (Fig. 1B). Therefore, melatonin and ethylene were primarily produced in preveraison berry skins, and MT treatment increased ethylene production. Additionally, 1-MCP was used to inhibit ethylene action, and its effects on ethylene production were determined in this study. Compared with the treatment of MT alone or the control, the application of 1-MCP in the presence or absence of MT did not exert significant effects on ethylene production (Fig. 1C). Therefore, the increases in ethylene production were attributed to MT in berry skins treated with MT + 1-MCP.

\section{Identification of differentially accumulated metabolites (DAMs) in response to MT and 1-MCP}

Berry skins collected at $110 \mathrm{DAB}$ were used to detect changes in metabolite concentrations in response to MT and the ethylene receptor inhibitor 1-MCP using the widely targeted metabolomics approach. High correlation coefficients (Fig. 2A) and different clustering (Fig. 2B) of three biological replicates for each treatment indicated the 


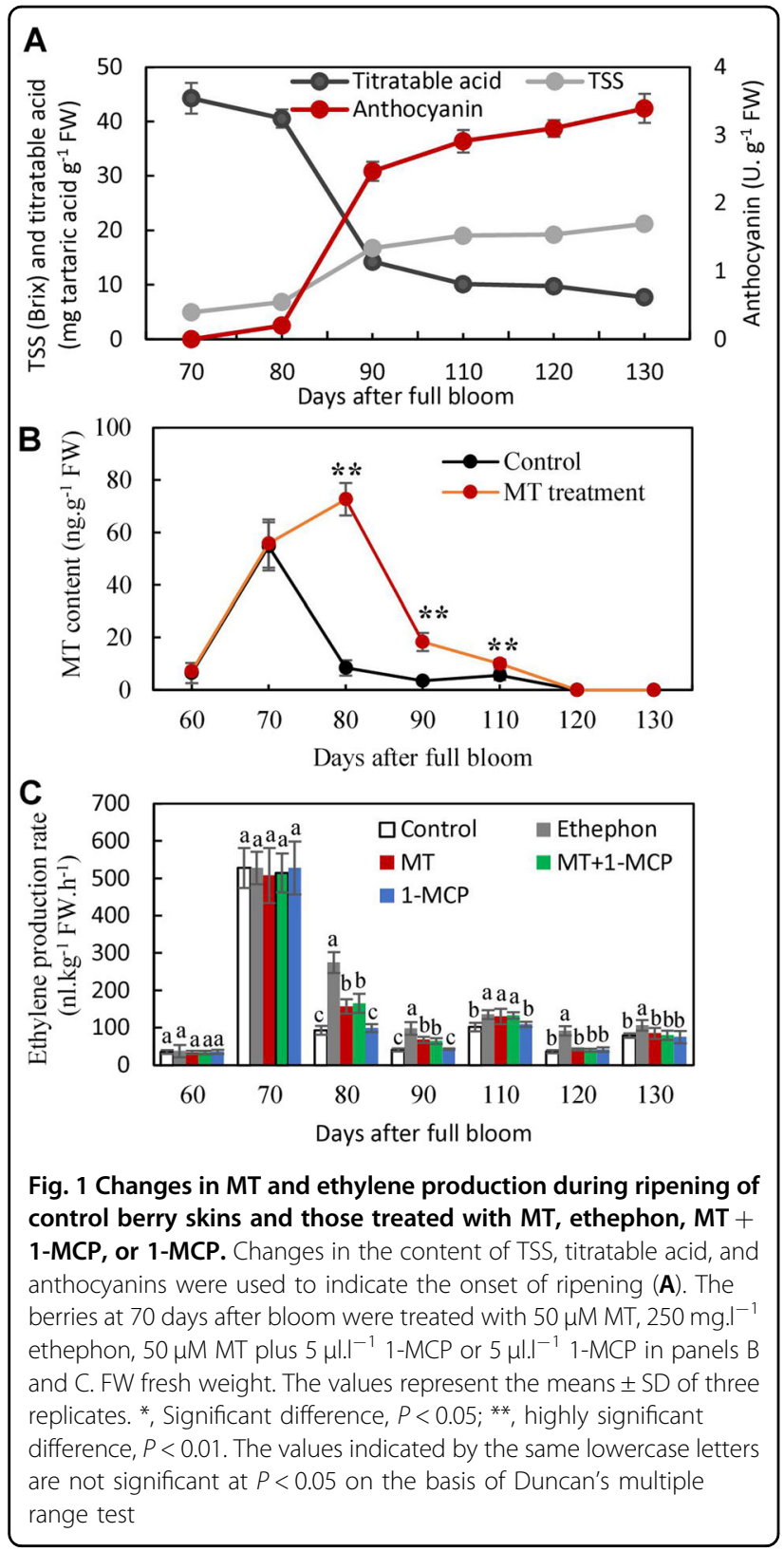

strong reliability of the generated metabolomic data and the large effects of the MT and MT_1-MCP (treatment with MT plus 1-MCP) treatments on metabolites. After quality validation, a total of 464 metabolites were detected in the control berry skins and in those treated with MT and MT_1-MCP (Table S2).

A total of 27 DAMs between pairs of treatments were identified on the basis of a fold change $\geq 2$ or $\leq 0.5$ and a VIP $\geq 1$. In total, 18 DAMs were identified between the control and MT groups, and only 4 DAMs were identified between the control and MT_1-MCP groups (Fig. 2C, D; Tables S3 and S4), indicating that inhibition of ethylene signaling by 1-MCP reduced the effects of MT on DAMs.
Additionally, 18 DAMs were identified in the MT_1-MCP vs MT comparison (Fig. 2E; Table S5). KEGG enrichment analysis showed that the DAMs in the control vs MT and the MT_1-MCP vs MT comparisons had very similar KEGG classifications. These included biosynthesis of secondary metabolites; stilbenoid, diarylheptanoid, and gingerol biosynthesis; and biosynthesis of flavonoids, flavones, and flavonols (Fig. 2F).

Additionally, the contents of the four metabolites in berry skins under different treatments were measured to verify the role of MT in regulating DAMs via ethylene (Fig. 2G). Compared to the control, ethephon treatment largely increased the content of transresveratrol and gallic acid and decreased the content of catechin and quercitrin; in contrast, 1-MCP application led to contrasting results; therefore, ethylene played a role in regulating the accumulation of these four metabolites. MT treatment caused similar effects to those caused by ethephon, and the application of 1-MCP caused significant inhibitory effects on MT-induced changes in the content of the four metabolites. Therefore, MT regulated the accumulation of the four metabolites via ethylene.

Additionally, the 27 DAMs were normalized and classified into six groups based on their changes in abundance in the different samples (Table 1). The abundance of 14 DAMs in groups 5 and 11 DAMs in groups 1, 4, and 6 increased and decreased in response to melatonin, respectively. In contrast, the changes in DAMs caused by MT were alleviated and even reversed by $1-\mathrm{MCP}$. Therefore, the regulatory effects of melatonin on the 25 DAMs were mediated by ethylene, at least in part.

\section{Identification of the changes in the transcriptome profile of berry skins in response to MT and 1-MCP}

RNA-seq analysis of the control skins and skins treated with MT and MT_1-MCP was conducted to quantify gene expression changes. High correlation coefficients (Fig. S1) and different clustering (Fig. 3A) of three biological replicates for each treatment indicated high reliability of the transcriptomic data. In total, the expression of 404 and 487 genes was significantly up- and downregulated, respectively, in the MT-treated skin compared with the control skin (Table S6). Furthermore, the expression of 410 and 655 genes was significantly up- and downregulated, respectively, in the MT-treated skin compared with the skins in the MT_1-MCP treatment (Table S7). The differentially expressed genes (DEGs) were classified into six groups according to the change patterns of the standardized FPKMs in the different samples (Fig. 3B; Table S8). Compared to the control, the MT treatment upregulated the expression levels of the genes in groups 5 and 6 ; however, the addition of 1-MCP reduced the extent of upregulated expression in group 5 and led to downregulated expression in group 6. In 


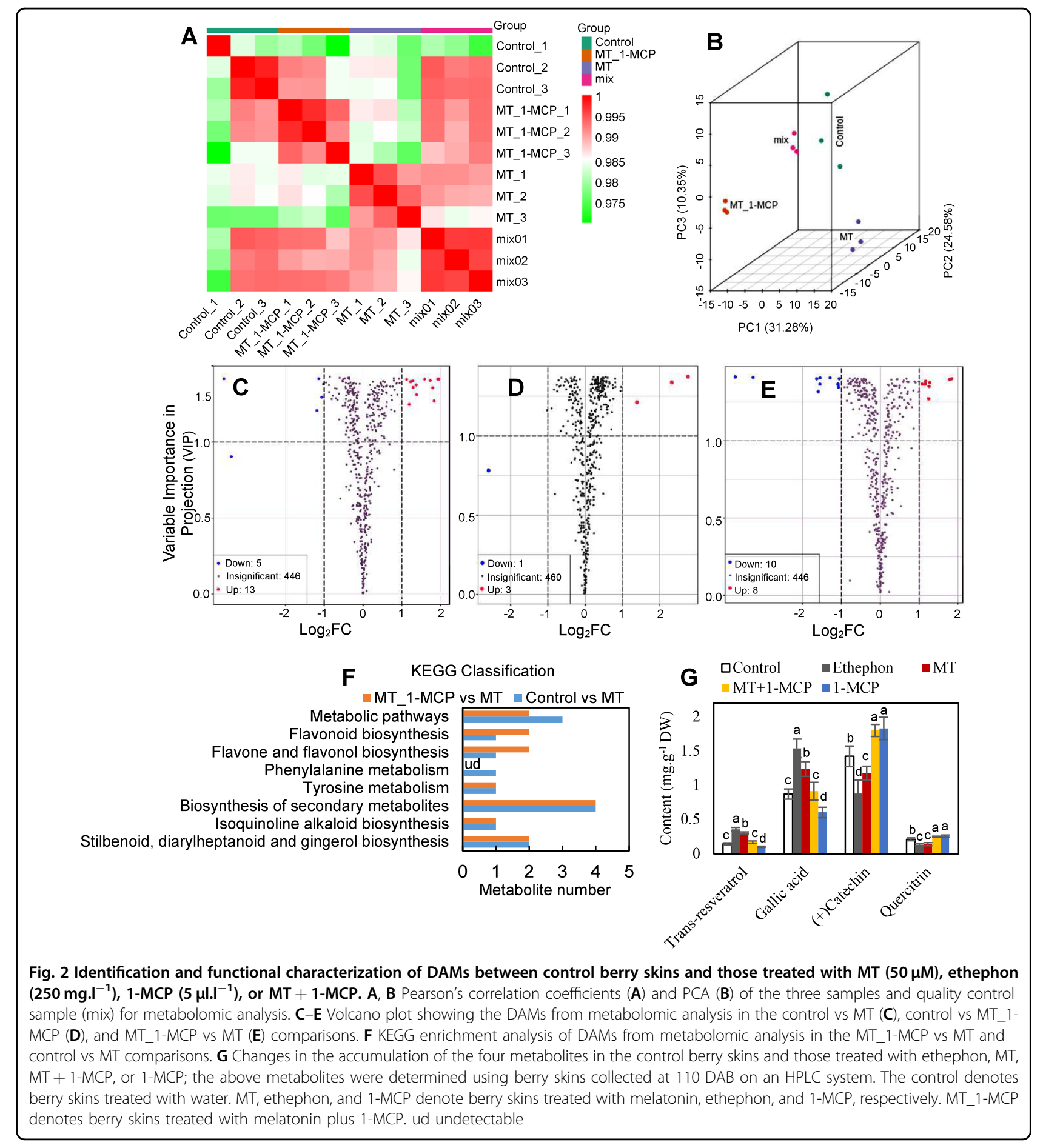

contrast, the opposite patterns were found for the genes in groups 1 and 4 under MT and 1-MCP treatment. Therefore, MT altered the expression of the above four groups of genes through ethylene to varying extents.

GO enrichment analysis showed that the DEGs in the control vs MT comparison group were primarily associated with secondary metabolism, including biosynthesis of phenylpropanoids, flavonoids, and proanthocyanidins, in terms of metabolic processes; in contrast, the DEGs in the MT_1-MCP vs MT comparison group were primarily related to photosynthesis and secondary metabolism (Fig. 3C). KEGG enrichment analysis indicated that the DEGs occurring simultaneously in the control vs MT and MT_1-MCP vs MT comparisons were primarily related to 
Table 1 Standardized intensity of DAMs in different samples

\begin{tabular}{|c|c|}
\hline Class & Compounds \\
\hline Flavonoids & $\begin{array}{l}\text { Delphinidin chloride, Luteolin 7-O-glucuronide, } \\
\text { Quercitrin, Avicularin, Kaempferol, } \\
\text { Quercetin 3,7-bis-O- } \beta \text {-D-glucoside }\end{array}$ \\
\hline Lipids & N-(2-hydroxyethyl)eicosapentaenoic acid \\
\hline $\begin{array}{l}\text { Amino acid } \\
\text { derivatives }\end{array}$ & S-methyl glutathione \\
\hline $\begin{array}{l}\text { Amino acid } \\
\text { derivatives }\end{array}$ & L-tyramine \\
\hline Others & Phenethylamine \\
\hline Phenolic acids & $\begin{array}{l}\text { Piceid, Pallidol, 3-(4-Hydroxyphenyl)-propionic acid, Piceatannol, 3-Hydroxy-4- } \\
\text { isopropylbenzylalcohol 3-glucoside-glucoside, Gallic acid }\end{array}$ \\
\hline Flavonoids & Propyl gallate \\
\hline Lipids & 13-HOTrE(r) \\
\hline Organic acids & DL-P-hydroxyphenyllactic acid \\
\hline Stilbene & Resveratrol, Pterostilbene \\
\hline Others & a-Viniferin, 9,10-Dihydrophenanthrene, Digalloylglucose \\
\hline Flavonols & Pinocembrin, Catechin, Kaempferin \\
\hline
\end{tabular}

\section{Control MT MT_1-MCP}
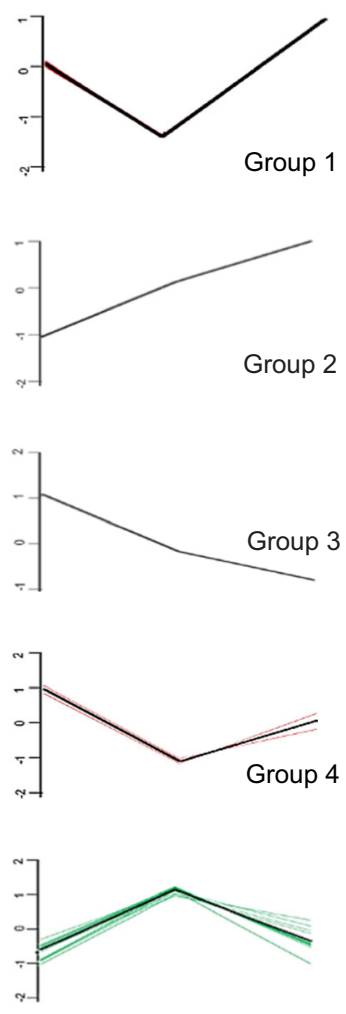

Group 5

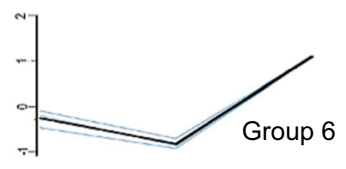

plant hormone signal transduction and biosynthesis of secondary metabolites, which included phenylpropanoid biosynthesis (Fig. 3D).

\section{Association analysis of DAMs and DEGs}

Nine quadrant diagrams were generated to systematically compare the variations in metabolites and their corresponding genes, with Pearson's correlation coefficients $>0.8$. The DAMs and DEGs shown in quadrants 1 and 9 were negatively associated, while the DAMs and DEGs shown in quadrants 3 and 7 were positively associated (Fig. 4A-C). Compared with those in the control vs MT comparison, the DAMs and DEGs in the control vs MT_1-MCP comparison largely decreased (Fig. 4A, B), indicating the role of ethylene in mediating MT signaling. Additionally, the MT_1-MCP vs
MT comparison showed that a total of 18 DAMs showed an association with DEGs, including 1191 genes in quadrants 3 and 7 and 1290 genes in quadrants 1 and 9 (Fig. 4C; Table S9). Moreover, stilbenoid, diarylheptanoid, and gingerol biosynthesis was the significantly changed KEGG pathway based on the DAMs and DEGs (Fig. 4D; Table S10). Further, to explore the relationship between the DAMs and DEGs, an O2PLS model was constructed, and the DEGs and DAMs were subsequently listed according to their distance to the center dot (Fig. 4E, F; Tables S11 and S12). In the X loading, 1-aminocyclopropane-1-carboxylate synthase 1 (ACS1), a key gene responsible for ethylene biosynthesis, was among the top 10 DEGs; additionally, three genes that encode transcription factors were in the top 50 DEGs: VvMYB14, $V v M Y B 86$, and ethylene-responsive transcription factor 

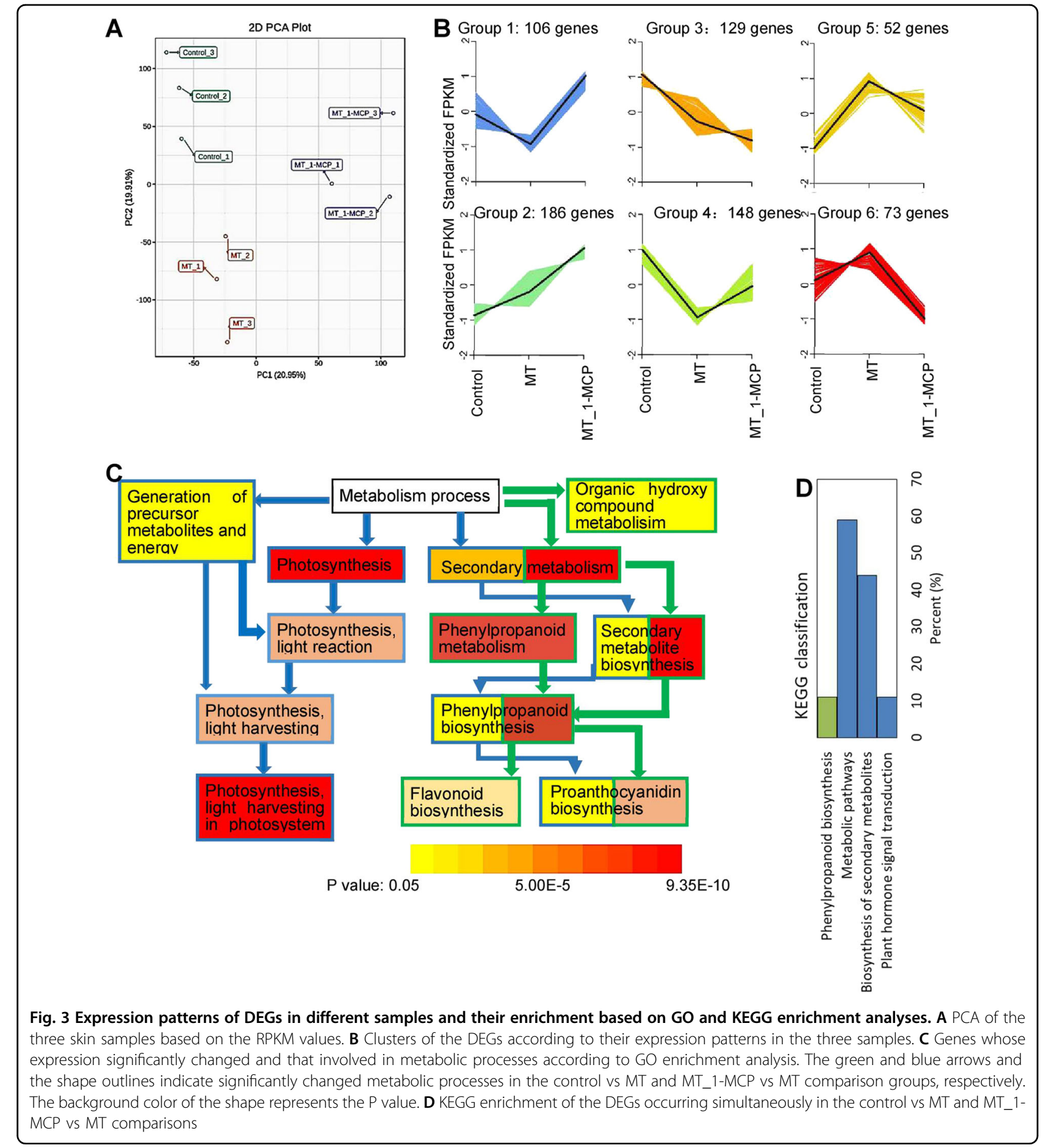

RAP2-2. VvMYB14 and VvACS1 were correlated with 17 and 15 DAMs (Table S11), respectively, suggesting their importance in regulating metabolites. In the $\mathrm{Y}$ loading, $\mathrm{N}-(2-$ hydroxyethyl) eicosapentaenoic acid, S-methyl glutathione, and pinocembrin were most strongly associated with the DEGs (Table S12).
To better understand the changes in metabolites induced by MT via ethylene, a proposed metabolic pathway with annotations of variations in metabolites and candidate genes was presented (Fig. 5). The secondary metabolites whose content changed primarily originated from phenylalanine (a precursor of phenylpropanoid 

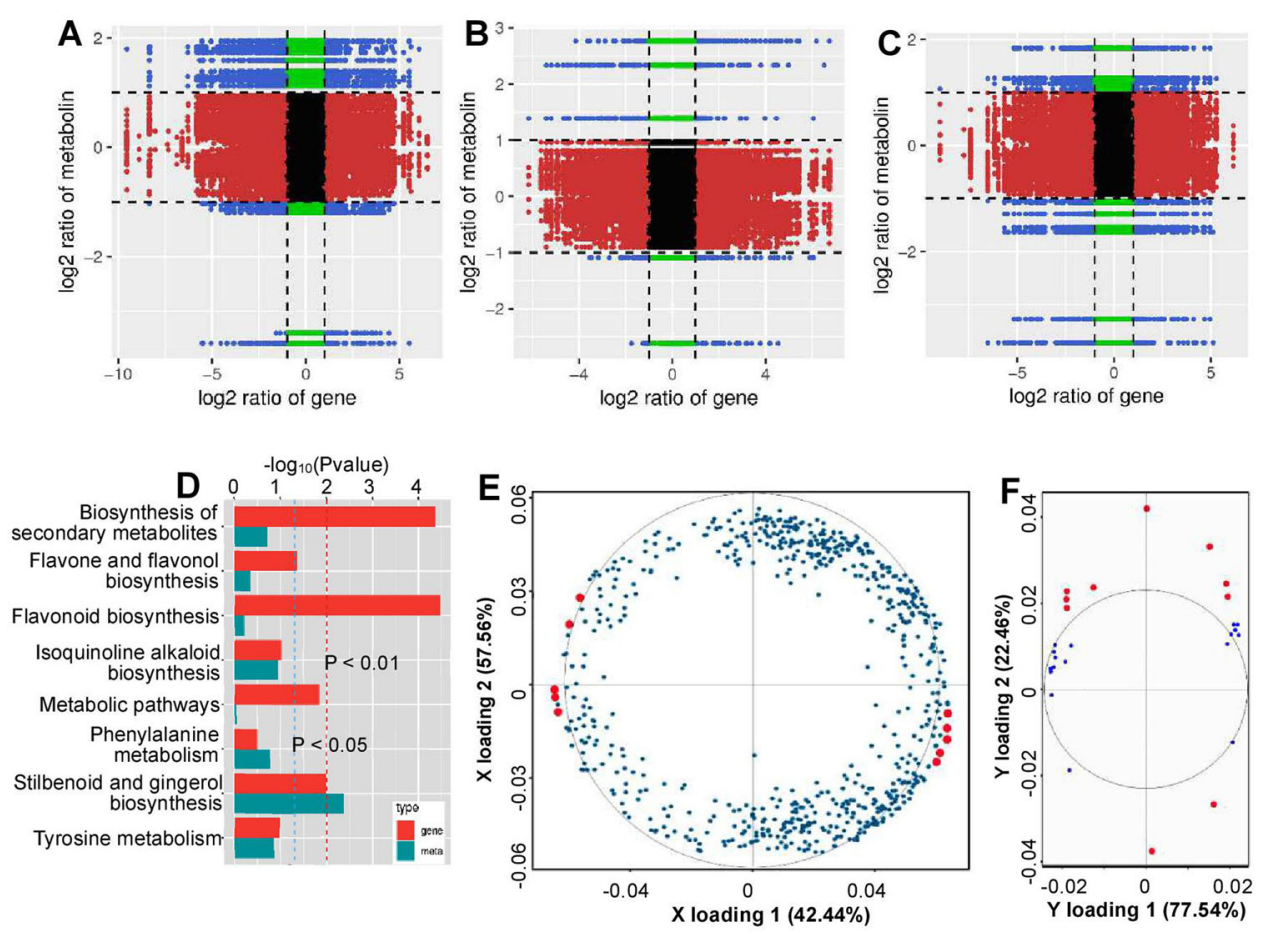

Fig. 4 Transcriptomic and metabolomic variation and their associations between pairs of treatments. A-C Overview of transcriptomic and metabolomic variation in the control vs MT (A), control vs MT_1-MCP (B), and MT_1-MCP vs MT (C) comparisons. The $x$-axis represents the fold changes of gene expression, and the $y$-axis represents the fold change of the metabolites. Each point represents a gene/metabolite. The black dots represent unchanged genes/metabolites, the green dots represent DAMs with unchanged genes, the red dots represent DEGs with unchanged metabolites, and the blue dots represent both DEGs and DAMs. D KEGG enrichment of DEGs and DAMs that are correlated with each other. E O2PLS model correlated component loadings for the DEGs. F O2PLS model correlated component loadings for the DAMs. A longer distance of the dots representing DEGs $(\mathbf{E})$ or DAMs $(\mathbf{F})$ to the center dot of the circle means a stronger correlation with DAMs $(\mathbf{F})$ or DEGs $(\mathbf{E})$. The ten most influential DEGs and DAMs are highlighted with red loading plots in panels $\mathbf{E}$ and $\mathbf{F}$, respectively

biosynthesis). The content of resveratrol and its four derivatives significantly increased in response to MT, and this increase was accompanied by the upregulation of STS1 expression; additionally, these increases were mediated by ethylene. The content of gallic acid and its two derivatives also significantly increased, and the expression of two shikimate dehydrogenases (SDHs) involved in gallic acid biosynthesis was significantly downregulated. In contrast, the content of tyramine and eight other metabolites (excluding phenethylamine) significantly decreased, which was mediated by ethylene. The expression of key genes involved in flavonoid biosynthesis, including F3Hs, LAR2, and DFR, was significantly downregulated, while that of FLS1 was significantly upregulated.

\section{MT induces the expression of the VvMYB14 gene, whose product binds to the promoter of VvACS1 and activates its transcription}

In our previous study, $V v A C S 1$ was proven to be a key gene controlling ACC biosynthesis and ethylene production $^{22}$. Additionally, ethylene production (Fig. 1B) and the expression of $V v A C S 1$ were induced by MT in berry skin (Fig. 6A, B). Therefore, $V v A C S 1$ functions in controlling ethylene production in response to MT in berry skin. The presence of the MBS element in the promoter of $V v A C S 1$ (Fig. S2) and expression association analysis (Fig. 6A, B; Fig. S3A) suggested the possible regulation of $V v A C S 1$ transcription by VvMYB14 and VvMYB86. Further yeast one-hybrid $(\mathrm{Y} 1 \mathrm{H})$ assays excluded the possibility of interactions of VvMYB86 and the VvACS1 promoter (Fig. S3B) and confirmed the binding of VvMYB14 to the MBS element within the $V v A C S 1$ promoter (Fig. 6C).

To further confirm this interaction, an electrophoretic mobility shift assay (EMSA) was performed using the purified VvMYB14 protein and a biotin-labeled MBS element (Fig. 6D). VvMYB14 bound to the $V v A C S 1$ promoter fragment containing MBS, and the binding was gradually reduced by the application of increasing amounts of unlabeled MBS competitor probe. In contrast, this competition was not detected when a mutated competitor was used. Therefore, the VvMYB14 protein specifically bound to the MBS element of the $V v A C S 1$ promoter. Additionally, the regulation of the $V v A C S 1$ 


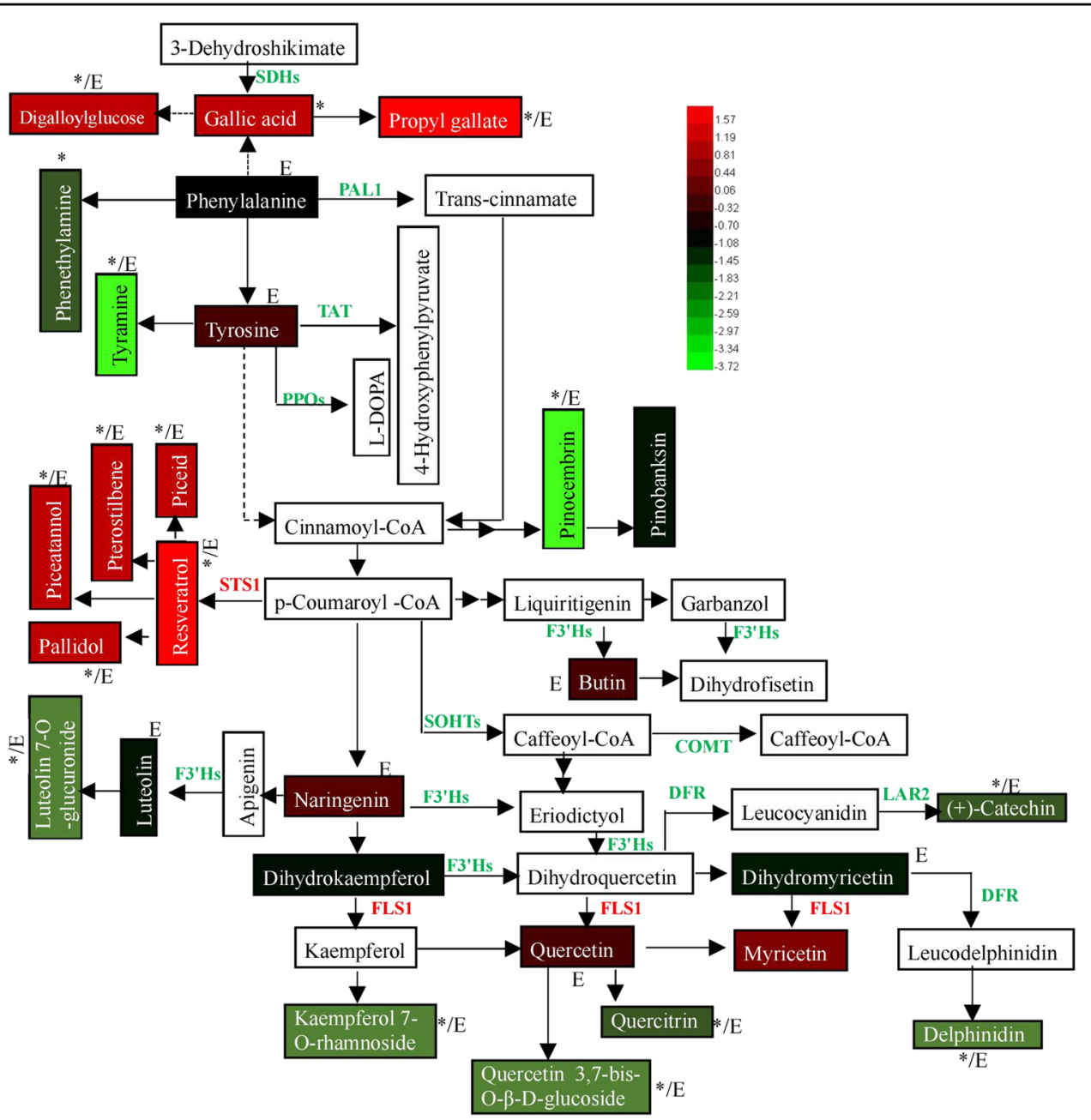

Fig. 5 KEGG pathway analysis of the metabolites and genes that changed in response to melatonin and/or ethylene. The schematic was made by integrating the pathways of ko00945, k000350, ko00360, ko00944, and ko00950. Each solid black arrow represents an enzyme-catalyzed reaction. The dotted line means that the uncertain enzyme-catalyzed processes are included. The boxes with white backgrounds indicate that metabolites were not detected in this study. The boxes with color represent the metabolites whose content changed in response to MT treatment, the difference in metabolite content is expressed as the $\log _{2}$ (fold change) value, and the normalized values are shown on a color scale. The asterisk represents a significant difference, and E means that inhibition of ethylene signaling by 1-MCP reduced the effects of MT on DAMs. The genes whose expression was upregulated or downregulated are highlighted with red or green colors, respectively. PAL1 phenylalanine ammonia lyase, VIT_206s0004g02620, TAT tyrosine aminotransferase, VIT_212s0028g03200, PPO polyphenol oxidase, VIT_200s0480g00070 and VIT_210s0116g00560, FLS1 flavonol synthase, VIT_218s0001g03470, STS1 stilbene synthase, VIT_216s0100g00950, F3'H flavonoid-3'-hydroxylase, VIT_217s0000g07200 and VIT_217s0000g07210, SOHT shikimate O-hydroxycinnamoyltransferase, VIT_202s0087g00370 and VIT_209s0096g00660, COMT caffeoyl-CoA Omethyltransferase, VIT_212s0028g03110, DFR dihydroflavonol 4-reductase, VIT_218s0001g12800, LAR2 leucoanthocyanidin reductase, VIT_217s0000g04150, SDH shikimate dehydrogenase, VIT_214s0030g00660 and VIT_214s0030g00650

promoter by VvMYB14 was determined using a $\beta$-glucuronidase (GUS) transactivation assay in tobacco leaves (Fig. 6E, F). Compared with tobacco leaves transformed with 35 S::MYB14 and the mutant Pacs1::GUS, tobacco leaves cotransformed together with $35 \mathrm{~S}: \mathrm{MYB14}$ and Pacs1::GUS constructs were more blue in color and showed higher GUS activity. Therefore, VvMYB14 increased $V v A C S 1$ promoter activity.

Additionally, the expression of $V v M Y B 14$ was strongly induced by MT (Fig. 6A, B). In particular, the transactivation ability of the $V v M Y B 14$ promoter was enhanced by MT, and a 580-bp region was demonstrated to be the core region responding to MT (Fig. 6G). Collectively, we inferred that MT promoted ethylene production by inducing the expression of $V v M Y B 14$ and therefore $V v A C S 1$.

\section{VvMYB14 mediates MT-induced ethylene production and modification of secondary metabolites in grape calli}

To verify the above inference, transgenic grape calli with different levels of $V v M Y B 14$ expression were obtained, 

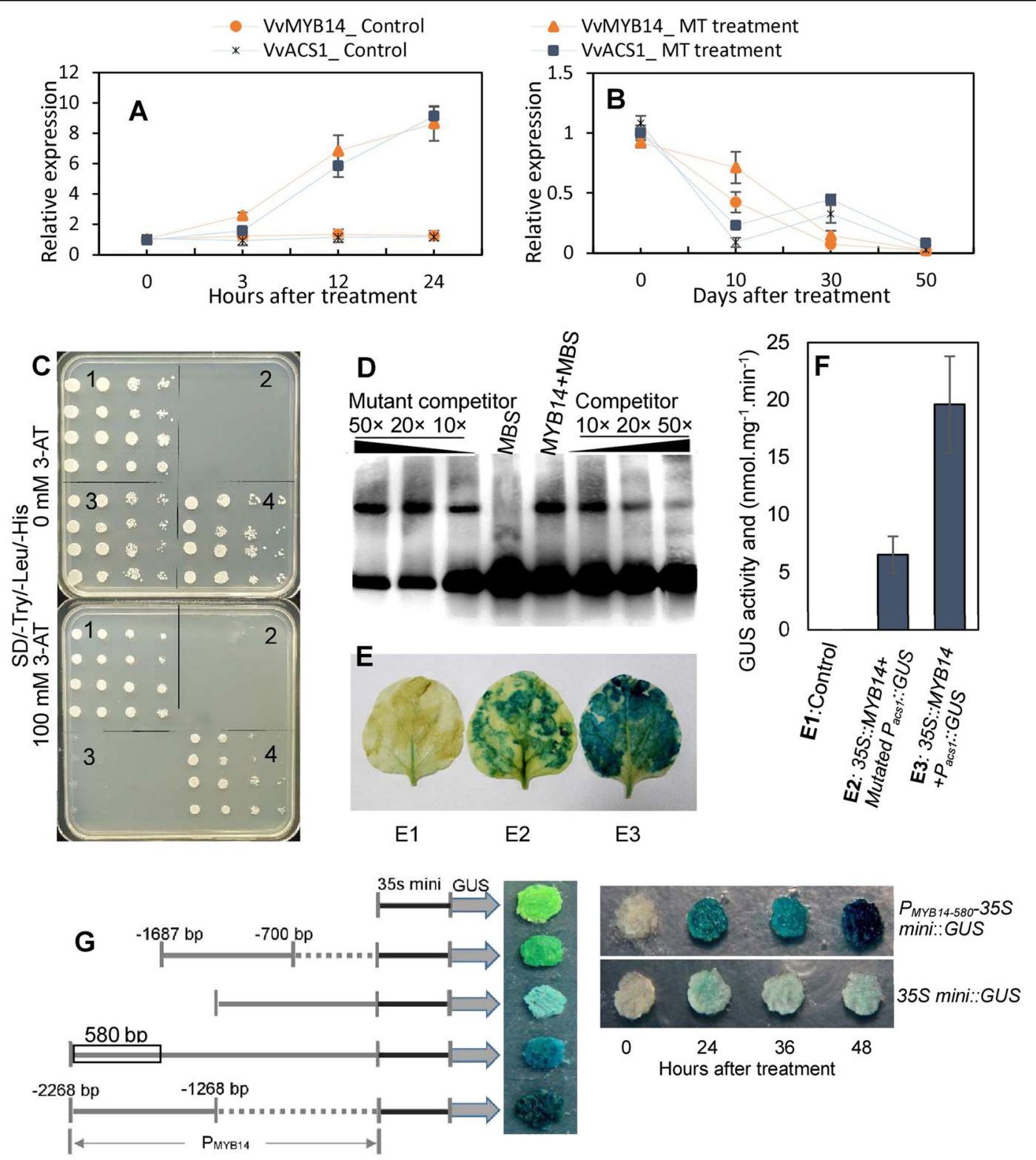

Fig. 6 Expression of $V v A C S 1$ and $V v M Y B 14$ in response to MT and characterization of the transcriptional activation of $V v A C S 1$ by VvMYB14. A, B MT treatment was applied at 70 DAB, and 50 days after treatment corresponds to the ripening stage of the grape berries in $\mathbf{B}$. C Yeast one-hybrid assay. (1) P53-pHis2 + pGADT7-REC2-53, Rec-P53 and the P53 promoter, whose interactions have been confirmed, acted as positive controls; (2) pHis2 + pGADT7. (3) MBS-pHis2 + pGADT7. (4) MBS-pHis2 + pGADT7-MYB14. In panels 1-4, the yeast cells were diluted 1-, 10-, 100-, and 1000-fold, respectively, from left to right. 3-AT (3-amino-1,2,4-triazole) was used as a screening marker ${ }^{54}$. D Interaction of the VvMYB14-His protein with the labeled DNA probes for MBS elements or mutant MBS elements within the VVACS1 promoter in an EMSA. E, F Histochemical staining (E) and GUS activity $(\mathbf{F})$ analysis of the transactivation activity of VVMYB14 by binding to the VVACS1 promoter. E1-E3 in panel $\mathbf{E}$ are annotated in panel $\mathbf{F}$. The 1500 bp sequence upstream of the start codon was used as the VVACS1 promoter, and the mutated VVACS1 promoter refers to the VVACS1 promoter sequence with the mutant MBS element that was the same as the mutant sequence used in the EMSA probe. G GUS staining of grape calli expressing the VVACS1 promoter fragment-35S mini::GUS construct. The VVACS1 promoter fragments are indicated by the gray solid lines, and the number denotes the length between the marked site and the start codon. PMYB14-580 represents the 580-bp fragment of the VVMYB14 promoter, marked with a black box

including five overexpression lines (OE1-5) and four suppression lines (SE1-4). Overexpression of $V v M Y B 14$ increased the expression levels of $V v A C S 1$, while suppression of $V v M Y B 14$ decreased $V v A C S 1$ expression
(Fig. 7A, B); additionally, the MT-induced increase in VvACS1 expression was largely reduced by VvMYB14 suppression (Fig. 7B). Therefore, MT enhanced VvACS1 expression via $V v M Y B 14$. 

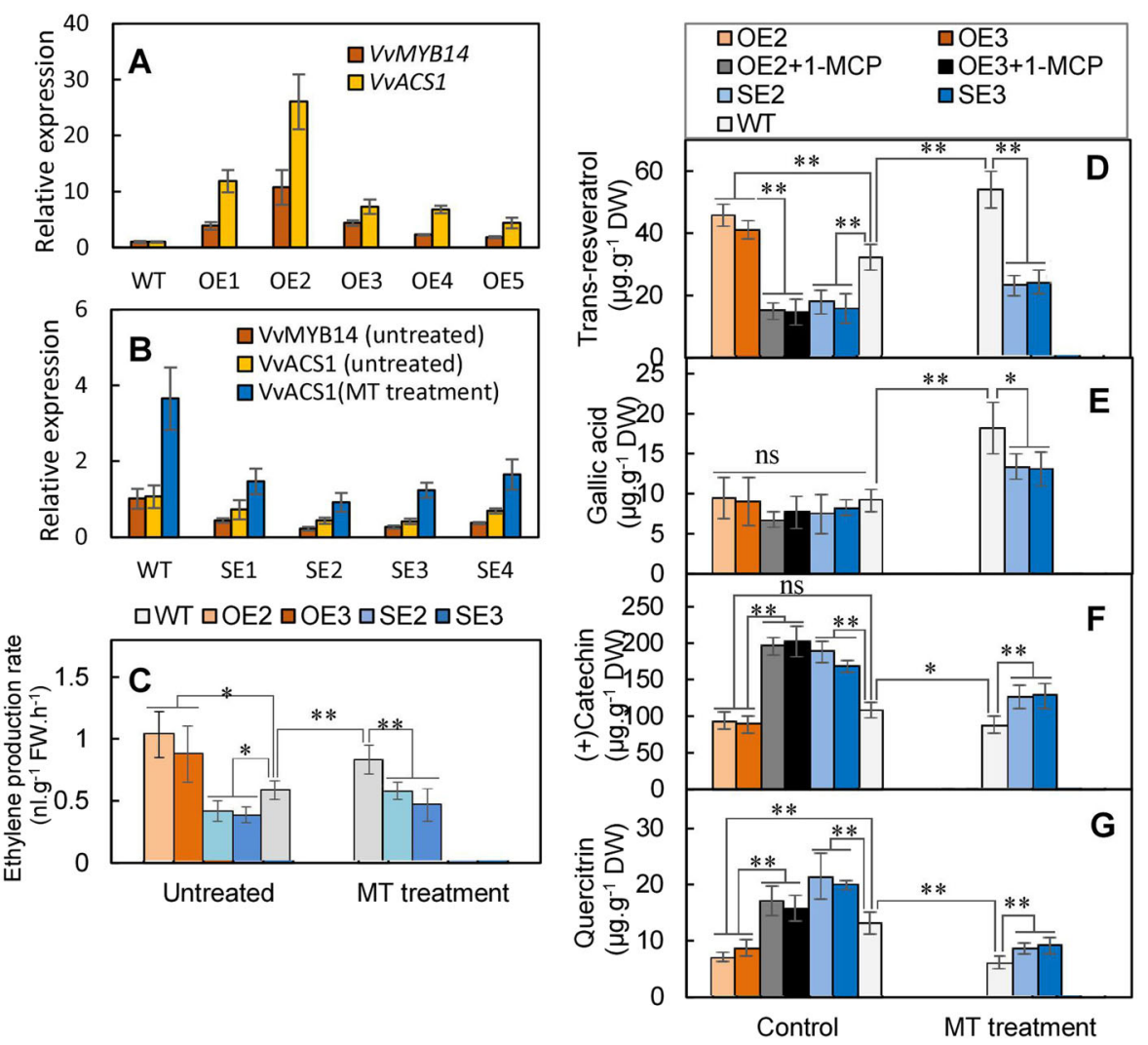

Fig. 7 Changes in ethylene production and accumulation of the four metabolites in VvMYB14-overexpressing and VvMYB14-suppressed grape calli in the presence or absence of MT. A, B Expression levels of VVMYB14 and VVACS1 in VVMYB14 overexpression lines (OEs) (A) and suppression lines (SEs) (B). C- G Ethylene production (C) and content of four metabolites (D- $\mathbf{G}$ ) in the two overexpression lines and two suppression lines in the presence or absence of MT. The OEs were treated with 1-MCP to evaluate the effects of the inhibition of ethylene signaling on the contents of the four metabolites

Additionally, two overexpression lines (OE2 and OE3) and two suppression lines (SE2 and SE3) with the highest and lowest expression levels of $V v M Y B 14$, respectively, were used to determine the effects of the $V v M Y B 14$ expression level on ethylene production and metabolite contents. $\quad V v M Y B 14$ overexpression significantly increased ethylene production, while its suppression led to the opposite results. MT increased ethylene production compared to that of the untreated control, and the MTinduced promotion of ethylene production was reduced in the suppression lines (Fig. 7C). Similar changes in transresveratrol content were found in the WT, overexpression lines and suppression lines; additionally, the transresveratrol content was largely reduced in response to $1-\mathrm{MCP}$ application in the overexpression lines (Fig. 7D). The gallic acid content significantly increased in response to MT, but the changes in the $V v M Y B 14$ expression level did not exert significant effects on the gallic acid content under the control conditions; however, VvMYB14 suppression reduced the MT-induced increase in gallic acid (Fig. 7E). In contrast, melatonin decreased the content of catechin and quercitrin, but overexpression and suppression of $V v M Y B 14$ decreased and increased their contents, respectively, in the presence or absence of MT treatment; additionally, the content of catechin and quercitrin largely increased in response to $1-\mathrm{MCP}$ in the overexpression lines (Fig. 7F, G). Collectively, MT at least partially promoted ethylene production via VvMYB14 and therefore altered the accumulation of metabolites.

\section{Discussion}

MT may influence ethylene production in berries in a skinspecific manner

The MT concentration in the Merlot berry skins decreased with increasing berry ripening (Fig. 1B). However, opposite trends have been observed in grape seed and flesh, where MT concentrations increased during ripening ${ }^{23}$. Therefore, different accumulation patterns of MT occur in the skin and in other berry tissues. Additionally, the ethylene release peak has been shown to occur before veraison not only in Merlot berry skin (Fig. 1B) but also in whole berries of Moldova and Cabernet 
Sauvignon ${ }^{5,24}$. In contrast, the peak MT content in the berry skin occurred at preveraison, which was consistent with the ethylene release peak (Fig. 1C); however, the MT peak occurred more than one month later than the ethylene release peak did in Moldova berries ${ }^{5}$. Therefore, different interplay mechanisms of ethylene and MT might occur in berry skin and in other berry tissues.

Increasing amounts of evidence have shown that MT promotes ethylene biosynthesis in fruits. In this study, MT treatment promoted ethylene production in berry skins (Fig. 1B); similar results were also found in Moldova berries $^{5}$. However, during tomato and banana postharvest ripening, MT treatment promotes and reduces ethylene production, respectively, through regulation of the expression of $A C O 1$ and $A C S 1^{12,14}$. Moreover, MT pretreatment decreased ethylene levels in alfalfa under waterlogging conditions ${ }^{25}$. A combination treatment of both MT and Fusarium wilt increased ethylene levels, whereas MT alone inhibited ethylene production in banana leaves ${ }^{26}$. The above opposite effects suggest that other regulators may mediate the interplay between MT and ethylene. This inference is supported by our previous study showing that MT promotes ethylene production in part via ABA during berry ripening ${ }^{13}$. Therefore, the effects of MT behavior on ethylene are complex and may largely depend on environmental factors, plant tissue and even developmental stage, and skin-specific interplay may occur in grape berries.

\section{Key secondary metabolic pathways modified by MT via ethylene in berry skins}

$1-\mathrm{MCP}$ is an ethylene action inhibitor that binds to the cellular ethylene receptor and effectively inhibits ethylene responses $^{8}$ and has been indicated to be a powerful tool for the inhibition of fruit ripening and senescence by blocking ethylene signaling in various nonclimacteric fruit crop species, including grape, cherry, citrus, and strawberry $^{27}$. In addition to these major effects, 1-MCP has differing and sometimes contradictory effects on fruit ethylene production; e.g., 1-MCP has no significant effects on ethylene production in grape berries (Fig. 1C) ${ }^{28}$ but inhibits ethylene production in peach fruits ${ }^{29}$. In the present study, 1-MCP was also used to inhibit ethylene signaling and evaluate the role of MT in modifying secondary metabolic pathways via ethylene. Changes in DAMs (groups 4 and 5 in Table 1 ) and DEGs (groups 4 and 5 in panel B of Fig. 3) caused by MT were alleviated by $1-\mathrm{MCP}$, indicating that MT functioned at least in part via ethylene. In contrast, 1-MCP treatment reversed the changes in DAMs (groups 1 and 6 in Table 1) and DEGs (groups 1 and 6 in panel B of Fig. 3) caused by MT. This may have occurred because these DAMs and DEGs were highly dependent on ethylene, and their changes were primarily regulated by ethylene rather than MT, which was supported by the similar increases in catechin and quercitrin in berry skins treated with MT $+1-\mathrm{MCP}$ and 1MCP compared to the control (Fig. 2G). Notably, compared with the MT treatment, the MT +1-MCP treatment produced additive effects on the DAMs (groups 2 and 3 in Table 1) and DEGs (groups 2 and 3 in panel B of Fig. 3), suggesting that these DAMs and DEGs may be antagonistically regulated by MT and ethylene and that the application of 1-MCP reduced the effects of ethylene, thereby improving the function of MT.

Resveratrol, gallic acid and their derivatives constituted the metabolites whose content significantly increased in response to MT via ethylene (Fig. 5). STS is the key enzyme responsible for the biosynthesis of resveratrol and its derivatives ${ }^{30}$, and the significant increase in resveratrol and its derivatives may be attributed to the upregulation of STS1 expression (Fig. 5). Additionally, the MT-induced increases in the content of resveratrol and its derivatives and the expression level of STS1 largely depend on ethylene (Fig. 5; Table S8), suggesting a role of ethylene in this process. In fact, ethylene has been reported to trigger the upregulation of stilbene biosynthesis-related gene expression and increase stilbene biosynthesis in grape and peanut $^{31,32}$. In particular, the large decrease in resveratrol in the $V v M Y B 14$-suppressed calli and in the calli treated with 1-MCP demonstrated the role of ethylene in mediating the regulation of resveratrol by MT (Fig. 7C, D). Additionally, our recent study revealed that MT increased the expression of another STS gene by decreasing its promoter methylation ${ }^{33}$. Therefore, STSs may represent a key point in the pathway regarding the regulation of resveratrol by MT or MT via ethylene.

Although it has long been recognized that plants, bacteria, and fungi synthesize and accumulate gallic acid, the pathway leading to its synthesis is largely unknown. Gallic acid biosynthesis from 3-dehydroshikimate, an intermediate in the shikimate pathway, has been reported ${ }^{34}$, and two grape shikimate dehydrogenases (VvSDH3 and VvSDH4) have been shown to be involved in gallic acid biosynthesis in grapevine ${ }^{35}$. However, in the present study, the expression levels of $V v S D H 3$ and $V v S D H 4$ were significantly downregulated in response to MT, although the content of gallic acid and its derivatives significantly increased (Fig. 5). Therefore, the shikimate pathway may not be the key pathway for gallic acid biosynthesis in berry skin under MT treatment. Additional studies are needed to reveal the MT-induced increase in gallic acid and its derivatives.

As shown in Fig. 5, the pathways mediated by F3' $\mathrm{H}$, DFR, and LAR2 may control the decreases in delphinidin, catechin, and luteolin 7-O-glucuronide contents under MT treatment. F3' $\mathrm{H}$ catalyzes the hydroxylation of the $3^{\prime}$ position of the B-ring of flavonoids, including naringenin and dihydrokaempferol. Mutation and downregulation of 
$F 3^{\prime} H$ result in compositional changes in flavonols ${ }^{36}$ and in delphinidin accumulation ${ }^{37}$, respectively. The expression of $F 3^{\prime} H$ and $F 3^{\prime} 5^{\prime} H$ genes directly affects the accumulation of anthocyanin compounds in grape berry $\operatorname{skin}^{38}$. DFR shows a preference for dihydroquercetin and dihydromyricetin as substrates to produce anthocyanins in cultivars of Vitis vinifera ${ }^{39}$. Therefore, decreases in the expression of $F 3^{\prime} H s$ and $D F R$ may reduce the synthesis of delphinidin, catechin, and luteolin and its derivatives by reducing the amount of their precursors. Additionally, LAR directly catalyzes the biosynthesis of catechin from leucocyanidin ${ }^{40}$, and the downregulation of $V v L A R 2$ expression directly contributes to a decrease in catechin. The synthesis of flavonol aglycones is catalyzed by FLS, which uses dihydroflavonols as substrates ${ }^{41}$. The increase in the expression of $V v F L S 1$ increased the contents of myricetin and quercetin, but they did not reach a significant level (Fig. 5). In contrast, the contents of kaempferol 7-O-rhamnoside, quercetin 3,7-bis-O- $\beta$-Dglucoside, and quercitrin significantly decreased, suggesting that other genes play a role. Here, the significant decrease in the content of the above compounds was dependent on ethylene, at least in part (Fig. 5; Tables S3-S5). However, various effects of ethylene on the content of secondary metabolites, including anthocyanins and catechin, have been reported ${ }^{42,43}$. Therefore, it is suggested that the regulation of secondary metabolites may be attributed to the combined effects of MT and ethylene in berry skin.

\section{VvMYB14 participates in the MT signaling pathway involved in the regulation of secondary metabolism}

Gene expression and promoter assays revealed the responses of $V v M Y B 14$ to MT (Fig. 6A, G). Additionally, the suppression of $V v M Y B 14$ reduced the effects of MT on ethylene and the detected secondary metabolites (Fig. 7C-G). Therefore, VvMYB14 participates in the MT signaling pathway. MYB14 may function in two ways in the MT signaling pathway. First, MYB14 responds to MT and directly activates gene expression. In grape, VvMYB14 and VvMYB15 are known to be involved in the transcriptional regulation of $V v S T S$ genes and to control stilbene levels in response to stresses ${ }^{19}$. MYB14 and MYB5 have been reported to regulate the proanthocyanidin pathway in seeds of $M$. truncatula, and their synergistic physical interactions increase the transcription of target proanthocyanidin pathway genes such as anthocyanidin reductase and anthocyanidin synthase $\mathrm{e}^{20,44}$.

Second, MYB14 may serve as a bridge between MT and other signaling molecules, thereby affecting secondary metabolism via other signaling molecules. A large number of studies in different species have shown that ethylene regulates the accumulation of secondary metabolites. For example, ethylene regulates anthocyanin and proanthocyanidin biosynthesis via the ethylene response factor MdERF1B in apple ${ }^{45}$, ethylene modulates flavonol accumulation in Arabidopsis ${ }^{46}$, and ethylene regulates polyphenol metabolism in grape berries ${ }^{47}$. Here, we revealed that VvMYB14 largely regulated secondary metabolism via ethylene signaling (Fig. 7). Additionally, Myb14 overexpression impacts the JA-related transcriptome and stimulates terpene and anthocyanin accumulation $^{48,49}$. The broad effects of MYB14 on gene expression and metabolites also suggest that MYB14 functions by regulating signaling pathways. For example, LjMYB14-overexpressing lotus plants show increased expression of genes involved in the general phenylpropanoid pathway and genes encoding enzymes of the isoflavonoid pathway ${ }^{50}$. Moreover, MYB14 has been identified as a putative regulator of a broad defense response involving flavonoids and isoprenoids in loblolly pine (Pinus taeda) $)^{51}$.

In summary, widely targeted metabolomics analysis revealed 27 DAMs whose abundance significantly changed in response to melatonin, of which the changes of 25 DAMs were mediated by ethylene. Transcriptome analyses indicated that melatonin primarily affects the pathways of plant hormone signal transduction and biosynthesis of secondary metabolites via ethylene. Association analysis of the DAMs and DEGs revealed that $F 3^{\prime} H \mathrm{~s}$, STS, DFR, and LAR2 play key roles in regulating DAMs under melatonin treatment. Additionally, melatonin induced the expression of VvMYB14, which increased ethylene production by transcriptionally activating $V v A C S 1$, thereby altering the accumulation of secondary metabolites.

\section{Materials and methods \\ Plant materials and growth conditions}

The present experiment was conducted at an experimental vineyard in Tai-An city, Shandong Province, China. Each vine had 10 vertical fruiting shoots on the horizontal cordon, and each fruiting shoot was controlled to produce a cluster. Preveraison Merlot grape (Vitis vinifera) berries at 70 days after full bloom (DAB) were subjected to MT treatment and 1-MCP treatment. The grape clusters on the vine were completely soaked for $5 \mathrm{~s}$ in a solution of $50 \mu \mathrm{M} \mathrm{MT}+0.05 \%$ Triton X-100, $50 \mu \mathrm{M}$ melatonin $+5 \mu \mathrm{ll}^{-1} 1-\mathrm{MCP}+0.05 \%$ Triton $\mathrm{X}-100$ or $250 \mathrm{mgl}^{-1}$ ethephon ${ }^{5}$. Treatment with $0.05 \%$ Triton X100 via the same method was used as a control ${ }^{5}$. Each treatment included three replications, and each replication comprised 6 vines. Approximately 180 berries from the shoulder, middle, and tail of each cluster at different days after treatment were collected for subsequent experiments. In addition, discs of tissue cut from Merlot berry skin were cultured on MS media comprising $0.1 \mathrm{mg}$ $\mathrm{l}^{-1} \mathrm{IBA}$ and $1.5 \mathrm{mgl}^{-1} \mathrm{TDZ}$ to induce nonembryogenic 
callus development. The obtained calli were subcultured on MS media comprising $0.59 \mathrm{gl}^{-1} 2$-(N-morpholino) ethanesulfonic acid, $10 \mathrm{mgl}^{-1}$ picloram, $2.2 \mathrm{mgl}^{-1}$ thidiazuron, $0.8 \mathrm{gl}^{-1}$ activated carbon, $30 \mathrm{gl}^{-1}$ sugar, and $7 \mathrm{gl}^{-1}$ agar at $25^{\circ} \mathrm{C}$ under dark conditions.

\section{Determination of anthocyanin, total soluble solids (TSS), and titratable acid contents}

The total anthocyanins in the berry skins were extracted and spectrophotometrically measured according to the methods described by $\mathrm{Xu}$ et $\mathrm{al}^{5}$. Fresh berry pulp was ground to a homogenate and filtered, and the filtrate was used for the determination of TSS and titratable acid contents. The TSS content was measured using a digitaldisplay sugar meter (PAL-1; Atago, Tokyo, Japan), and the titratable acid content was determined by titration of the filtrate with $0.1 \mathrm{M} \mathrm{NaOH}$ to an endpoint, at $\mathrm{pH}$ 8.3.

\section{Determination of the MT content and ethylene production rate}

MT was extracted and determined according to the methods of $\mathrm{Xu}$ et $\mathrm{al}^{5}$. The primary extraction procedures included preliminary extraction via an ultrasonic bath in methanol, evaporation of the extraction solution to dryness, and purification of the extract using a $\mathrm{C}_{18}$ solidphase extraction cartridge (ProElut ${ }^{\mathrm{TM}}$; Dikma, China). MT was determined using an UHPLC-MS system in conjunction with an ACQUITY UHPLC system and a QTOF micro-mass spectrometer (Waters, Milford, MA, USA). The parameters were as follows: mobile phase, $0.05 \%(\mathrm{v} / \mathrm{v})$ acetic acid and methanol at $0.3 \mathrm{ml} \mathrm{min}^{-1}$; column temperature, $25^{\circ} \mathrm{C}$; capillary temperature, $300{ }^{\circ} \mathrm{C}$; spray voltage, $3000 \mathrm{~V}$; auxiliary pressure, $15 \mathrm{~V}$; and sheath pressure, $35 \mathrm{~V}$.

Five grams of berry skin was enclosed in a $100-\mathrm{mL}$ jar and incubated for $3 \mathrm{~h}$ at $25^{\circ} \mathrm{C}$. Five milliliters of the headspace gas was then withdrawn from each jar using an air-tight syringe for ethylene determination. The ethylene concentration was determined using a GC-9A gas chromatograph (Shimadzu, Kyoto, Japan). The ethylene production rate was calculated on the basis of the ethylene concentration, incubation time, and skin weight ${ }^{5}$.

\section{Widely targeted metabolomics analysis}

Metabolome extraction and analysis were performed by a commercial company (Metware Biotechnology Co., Ltd., Wuhan, China). In brief, the lyophilized berry skin was ground in a mixer mill (MM 400, Retsch) and then extracted with $70 \%$ methanol, followed by absorption (CNWBOND Carbon-GCB SPE Cartridge) and filtration (SCAA-104, $0.22 \mu \mathrm{m}$ pore size) (ANPEL, Shanghai, China). The filtrate was analyzed using a UPLC-ESI-MS/ MS system in conjunction with UPLC (Shim-pack UFLC Shimadzu CBM30A system) and MS (Applied Biosystems
4500 Q TRAP). The UPLC and MS conditions were set according to the methods described by Guo et $\mathrm{al}^{52}$. The metabolites were identified using the Metware database (MWDB). The metabolite abundances were quantified according to their peak areas. Metabolites were considered to have differentially accumulated when the variable importance in projection (VIP) was $\geq 1$ and the absolute $\log _{2}$ (fold change) was $\geq 1$.

\section{Extraction and determination of the four metabolites}

Transresveratrol, gallic acid, catechin, and quercitrin were extracted and determined according to the methods of $\mathrm{Xu}$ et al. $^{13}$ and Sun et al. ${ }^{53}$. The primary extraction process included ultrasonication in a methanol solution, filtration, evaporation of the filtrate to dryness, and dissolving of the residue in chromatography-grade methanol. The determination of the metabolites was performed on an HPLC system (Waters 600, Waters, Milford, MA, USA). Resveratrol was isolated via gradient elution from $5 \%(\mathrm{~V} / \mathrm{V})$ acetonitrile to $75 \%$ acetonitrile and then measured at $307 \mathrm{~nm}$. The other three phenolics were isolated as follows: $90 \% \mathrm{~A}$ (water:acetic acid, 98:2) and 10\% B (acetonitrile) for $30 \mathrm{~min}, 65 \% \mathrm{~A}$ and 35\% B for $42 \mathrm{~min}$, and then $90 \% \mathrm{~A}$ and $10 \% \mathrm{~B}$ for $45 \mathrm{~min}$. The signal was monitored at $280 \mathrm{~nm}$.

\section{RNA-seq and quantitative RT-PCR}

Sequencing libraries were constructed using a NEBNext $^{\circledR}$ Ultra ${ }^{\text {TM }}$ RNA Library Prep Kit for Illumina ${ }^{\circledR}$ (\#7530 L, NEB, USA) according to the manufacturer's instructions. The libraries were sequenced on an Illumina HiSeq 4000 platform after a series of preparatory procedures, primarily those involving determinations of the RNA concentration and insert size and clustering of the index-coded samples. One hundred fifty-base pair pairedend reads were generated, and the clean reads were assembled into transcripts using Cufflinks, with the grape genome (http://genomes.cribi.unipd.it/grape/) used as a reference. Reads per fragment per kilobase of transcript per million mapped reads (RPKM) were used to quantify unigene expression levels, and the DEGs were screened in accordance with the following criteria: false discovery rate $<0.05$ and absolute $\log _{2}$ (fold change) $\geq 1$. Real-time quantitative PCR was performed using SYBR Green Master Mix (SYBR Premix EX Taq ${ }^{\mathrm{TM}}$, Dalian, China) on an ABI7500 qRT-PCR instrument (ABI, MA, USA), and the primers used are listed in Table S1.

\section{Yeast one-hybrid assays and electrophoretic mobility shift assays (EMSAs) \\ Yeast one-hybrid assays were conducted using a} Matchmaker $^{\mathrm{TM}}$ Gold Yeast One-Hybrid Library Screening System (Clontech, Mountain View, CA, USA). The sequence including the underlined MBS element 
(TACCCTCTCATGTCCCTGTGAACCTAACGTAAGG CATTACGATTTGTAT) from the promoter of $V v A C S 1$ was synthesized and inserted into a pHis2 vector. The ORF of $V v M Y B 14$ was subsequently amplified and inserted into a pGADT7 vector. The resultant plasmid was introduced into the yeast strain Y1HGold. The detailed procedure was performed according to the user manual for this system, and 3-AT was used as a screening marker ${ }^{54}$.

For the EMSA experiment, the VvMYB14-His recombinant protein was obtained using a pEASY-E1 expression vector (TransGen Biotech, Beijing, China) and purified using His-tagged BeaverBeads ${ }^{\mathrm{TM}}$ IDA-Nickel (Beaver, BioBay, China). Oligonucleotide probes containing an MBS element (CATGTCCCTGTGAACCTAACGTAAG GCA) and a mutant probe (CATGTCCCTGTGTACATA TCGTAAGGCA) were synthesized and labeled with biotin (Sangon, Shanghai, China). EMSAs were performed as described in the instruction manual included with the EMSA kit (Thermo Fisher Scientific, MA, USA) used. All the primers used are listed in Table S1.

\section{Transient cotransformation in tobacco leaves}

The VvMYB14 ORF was cloned and ligated into a pRI101-AN vector (Takara, Dalian, China) downstream of the $35 S$ promoter, yielding a $35 S:: M Y B 14$ plasmid. The $V v A C S 1$ promoter, the region up to 1500 bp upstream of ATG, and its mutated form with the mutant MBS element were used to replace the $35 S$ promoter within pRI101GUS, yielding a Pacs1::Gus plasmid and a mutant Pacs1:: Gus plasmid. The plasmids were subsequently introduced into Agrobacterium strain GV3101. The Agrobacteriummediated transient transformation of tobacco leaves was performed according to the methods of Yang et $\mathrm{al}^{55}$. GUS histochemical staining and activity detection were then performed according to the methods of Jefferson et al. ${ }^{56}$.

\section{Transformation of $V v M Y B 14$ into Merlot grape calli}

The abovementioned 35 S::MYB14 construct was used for sense overexpression. The $3^{\prime}$-UTR sequence of $V v M Y B 14$ was cloned into a pRI101-AN vector for antisense suppression. The $V v M Y B 14$ promoter fragments were isolated and inserted upstream of $35 \mathrm{~S}$ mini-GUS. The resultant constructs were introduced into Agrobacterium strain LBA4404, which was then transformed into grape calli according to a previous method ${ }^{22}$. The primers used in this experiment are listed in Table S1.

\section{Statistical analysis}

Principal component analysis (PCA) was performed, and Pearson correlation coefficients (PCCs) were calculated using the statistical function prcomp and the cor function of R software (base package, v3.5.0). Orthogonal projections to latent structures-discriminant analysis
(OPLS-DA) and variable importance in projection (VIP) values were generated using the $R$ package MetaboAnlystR. Fisher's exact test was applied to identify the significant KEGG pathways that had a false discovery rate $(\mathrm{FDR})<0.05$. Analysis of variance (ANOVA) and significant difference tests were performed using SPSS (v19.0) software.

\section{Acknowledgements}

This study was financially supported by the National Key R\&D Program of China (2018YFD1000200), the Key Research and Development Program of Shandong Province (2019GNC106149), the National Natural Science Foundation of China (31872068), the Funds of Shandong "Double Tops" Program (SYL2017YSTD10), and Shandong Province Government (SDAIT-06-03).

\section{Author contributions}

Y.Y. and W.M. conceived and designed the research; W.M., L.X., and X.L. performed the experiments; S.G. and X.C. analyzed the data; and Y.Y. wrote the manuscript. All the authors have read and approved the manuscript.

\section{Data availability}

The full RNA-seq data have been submitted to the Sequence Read Archive (SRA) of the NCBI under BioSample accession PRJNA646043 (https://www.ncbi. nlm.nih.gov/sra).

\section{Conflict of interest}

The authors declare that they have no conflict of interest.

Supplementary information The online version contains supplementary material available at https://doi.org/10.1038/s41438-021-00478-2.

Received: 2 September 2020 Revised: 7 December 2020 Accepted: 26 December 2020

Published online: 01 March 2021

\section{References}

1. Ali, K., Maltese, F., Choi, Y. H. \& Verpoorte, R. Metabolic constituents of grapevine and grape-derived products. Phytochem. Rev. 9, 357-378 (2010).

2. Yang, M. et al. Exogenous melatonin and abscisic acid expedite the flavonoids biosynthesis in grape berry of Vitis vinifera cv. Kyoho. Molecules 25, 12 (2019).

3. Fortes, A. M., Teixeira, R. T. \& Agudeloromero, P. Complex interplay of hormonal signals during grape berry ripening. Molecules 20, 9326-9343 (2015).

4. Lannetta, P. P. et al. Ethylene and carbon dioxide production by developing strawberries show a correlative pattern that is indicative of ripening climacteric fruit. Physiol. Plant. 127, 247-259 (2006).

5. Xu, L., Yue, Q., Xiang, G., Bian, F. \& Yao, Y. Melatonin promotes ripening of grape berry via increasing the levels of $\mathrm{ABA}, \mathrm{H}_{2} \mathrm{O}_{2}$, and particularly ethylene. Hortic. Res. 5, 1-11 (2018).

6. Aizat, W. M. et al. Metabolomics of capsicum ripening reveals modification of the ethylene related-pathway and carbon metabolism. Postharvest Biol. Tec. 89, 19-31 (2014).

7. Huber, D. J. Suppression of ethylene responses through application of 1methylcyclopropene: a powerful tool for elucidating ripening and senescence mechanisms in climacteric and nonclimacteric fruits and vegetables. HortScience 43, 106-111 (2008).

8. Sisler, E. C. \& Serek, M. Inhibitors of ethylene responses in plants at the receptor level: recent developments. Physiol. Plant 100, 577-582 (1997).

9. Liu, M. et al. The effects of light and ethylene and their interaction on the regulation of proanthocyanidin and anthocyanin synthesis in the skins of Vitis vinifera berries. Plant Growth Regul. 79, 377-390 (2016).

10. Serradilla, M. J., Falagan, N., Bohmer, B., Terry, L. A. \& Alamar, M. C. The role of ethylene and 1-MCP in early-season sweet cherry 'Burlat' storage life. Sci. Hortic. 258, 108787 (2019)

11. Arnao, M. B. \& Hernándezruiz, J. Melatonin: a new plant hormone and/or a plant master regulator? Trends Plant Sci. 24, 38-48 (2019).

12. Sun, Q. et al. Melatonin promotes ripening and improves quality of tomato fruit during postharvest life. J. Exp. Bot. 66, 657-668 (2015). 
13. $\mathrm{Xu}$, L. et al. Melatonin enhances phenolics accumulation partially via ethylene signaling and resulted in high antioxidant capacity in grape berries. Front. Plant Sci. 8, 1426 (2017).

14. $\mathrm{Hu}, \mathrm{W}$. et al. Natural variation in banana varieties highlights the role of melatonin in postharvest ripening and quality. J. Agr. Food Chem. 65, 9987-9994 (2017).

15. Aghdam, M. S. \& Fard, J. R. Melatonin treatment attenuates postharvest decay and maintains nutritional quality of strawberry fruits (Fragariaxanannasa cV. Selva) by enhancing GABA shunt activity. Food Chem. 221, 1650-1657 (2017).

16. Zhang, $Y$. et al. Delay of postharvest browning in litchi fruit by melatonin via the enhancing of antioxidative processes and oxidation repair. J. Agr. Food Chem. 66, 7475-7484 (2018).

17. Liu, J. et al. Effects of exogenous application of melatonin on quality and sugar metabolism in 'Zaosu' pear fruit. J. Plant Growth Regul. 38, 1161-1169 (2019).

18. Arnao, M. B. \& Hernandezruiz, J. Melatonin and its relationship to plant hormones. Ann. Bot. 121, 195-207 (2018).

19. Holl, J. et al. The R2R3-MYB transcription factors MYB14 and MYB15 regulate stilbene biosynthesis in Vitis vinifera. Plant Cell 25, 4135-4149 (2013).

20. Liu, C., Jun, J. \& Dixon, R. A. MYB5 and MYB14 play pivotal roles in seed coat polymer biosynthesis in Medicago truncatula. Plant Physiol. 165, 1424-1439 (2014).

21. Xiao, Q. et al. ZmMYB14 is an important transcription factor involved in the regulation of the activity of the $\mathrm{ZmBT1}$ promoter in starch biosynthesis in maize. FEBS J. 284, 3079-3099 (2017).

22. $\mathrm{Xu}, \mathrm{L}$. et al. Melatonin enhances salt tolerance by promoting MYB108Amediated ethylene biosynthesis in grapevines. Hortic. Res. 6, 1-14 (2019).

23. Vitalini, S. et al. The presence of melatonin in grapevine (Vitis vinifera L.) berry tissues. J. Pineal Res. 51, 331-337 (2011).

24. Chervin, $C$. et al. Ethylene seems required for the berry development and ripening in grape, a non-climacteric fruit. Plant Sci. 167, 1301-1305 (2004).

25. Zhang, Q. et al. Melatonin improved waterlogging tolerance in Alfalfa (Medicago sativa) by reprogramming polyamine and ethylene metabolism. Front. Plant Sci. 10, 44 (2019)

26. Wei, Y. et al. Identification, transcriptional and functional analysis of heat-shock protein 90 s in banana (Musa acuminate L.) highlight their novel role in melatoninmediated plant response to Fusarium wilt. J. Pineal Res. 62, e12367 (2017).

27. Li, L. et al. Effects of the ethylene-action inhibitor 1-methylcyclopropene on postharvest quality of non-climacteric fruit crops. Postharvest Biol. Technol. 111, 322-329 (2016).

28. Li, L. et al. The effects of 1-methylcyclopropene and ethylene on postharvest rachis browning in table grapes. Postharvest Biol. Technol. 107, 16-22 (2015).

29. Wu, Z. Q., An, X. J., Yu, M. L., Ma, R. J. \& Yu, Z. F. 1-Methylcyclopropene treatment on phenolics and the antioxidant system in postharvest peach combined with the liquid chromatography/mass spectrometry technique. J. Agric. Food Chem. 66, 6364-6372 (2018)

30. Lanz, T., Tropf, S., Marner, F. J., Schroder, J. \& Schroder, G. The role of cysteines in polyketide synthases. Site-directed mutagenesis of resveratrol and chalcone synthases, two key enzymes in different plant-specific pathways. J. Biol. Chem. 266, 9971-9976 (1991).

31. Belhadj, A. et al. Ethephon elicits protection against Erysiphe necator in grapevine. J. Agr. Food Chem. 56, 5781-5787 (2008).

32. Chung, I., Park, M. R., Rehman, S. U. \& Yun, S. J. Tissue specific and inducible expression of resveratrol synthase gene in peanut plants. Mol. Cells 12 353-359 (2001)

33. Gao, S., Ma, W., Lyu, X., Cao, X. \& Yao, Y. Melatonin may increase disease resistance and flavonoid biosynthesis through effects on DNA methylation and gene expression in grape berries. BMC Plant Biol. 20, 231 (2020).

34. Ossipov, V., Salminen, J., Ossipova, S., Haukioja, E. \& Pihlaja, K. Gallic acid and hydrolysable tannins are formed in birch leaves from an intermediate compound of the shikimate pathway. Biochem. Syst. Ecol. 31, 3-16 (2003).

35. Bontpart, $T$. et al. Two shikimate dehydrogenases, VvSDH3 and VvSDH4, are involved in gallic acid biosynthesis in grapevine. J. Exp. Bot. 67, 3537-3550 (2016).
36. Ryan, K. G., Swinny, E. E., Winefield, C. \& Markham, K. R. Flavonoids and UV photoprotection in Arabidopsis mutants. Z. Naturforsch. C. 56 745-754 (2001).

37. Huang, H., Hu, K., Han, K., Xiang, Q. \& Dai, S. Flower colour modification of chrysanthemum by suppression of $\mathrm{F}^{\prime} \mathrm{H}$ and overexpression of the exogenous Senecio cruentus F3'5'H gene. PLOS ONE 8, e74395 (2013).

38. Castellarin, S. D. et al. Colour variation in red grapevines (Vitis vinifera L.): genomic organisation, expression of flavonoid 3'-hydroxylase, flavonoid 3',5'-hydroxylase genes and related metabolite profiling of red cyanidin-/blue delphinidin-based anthocyanins in berry skin. BMC Genomics 7, 12 (2006)

39. Xie, S., Zhao, T., Zhang, Z. \& Meng, J. Reduction of dihydrokaempferol by Vitis vinifera dihydroflavonol 4-reductase to produce orange pelargonidin-type anthocyanins. J. Agr. Food Chem. 66, 3524-3532 (2018).

40. Pang, Y., Peel, G. J., Wright, E., Wang, Z. \& Dixon, R. A. Early steps in proanthocyanidin biosynthesis in the model legume Medicago truncatula. Plant Physiol. 145, 601-615 (2007).

41. Czemmel, S. et al. The grapevine R2R3-MYB transcription factor VVMYBF1 regulates flavonol synthesis in developing grape berries. Plant Physiol. 151, 1513-1530 (2009).

42. Jeong, S. W. et al. Ethylene suppression of sugar-induced anthocyanin pigmentation in Arabidopsis. Plant Physiol. 154, 1514-1531 (2010).

43. Ke, S., Chen, G., Chen, C., Tzen, J. T. \& Yang, C. Ethylene signaling modulates contents of catechin and ability of antioxidant in Camellia sinensis. Bot. Stud. 59, 1-8 (2018).

44. Verdier, J. et al. MtPAR MYB transcription factor acts as an on switch for proanthocyanidin biosynthesis in Medicago truncatula. PNAS 109, 1766-1771 (2012).

45. Zhang, J. et al. The ethylene response factor MdERF1B regulates anthocyanin and proanthocyanidin biosynthesis in apple. Plant Mol. Biol. 98, 205-218 (2018).

46. Lewis, D. R. et al. Auxin and ethylene induce flavonol accumulation through distinct transcriptional networks. Plant Physiol. 156, 144-164 (2011).

47. Becatti, E., Genova, G., Ranieri, A. \& Tonutti, P. Postharvest treatments with ethylene on Vitis vinifera (cv Sangiovese) grapes affect berry metabolism and wine composition. Food Chem. 159, 257-266 (2014).

48. Bedon, F. et al. Subgroup 4 R2R3-MYBs in conifer trees: gene family expansion and contribution to the isoprenoid- and flavonoid-oriented responses. J. Exp. Bot. 61, 3847-3864 (2010).

49. Zhang, $K$. et al. Jasmonate-responsive MYB factors spatially repress rutin biosynthesis in Fagopyrum tataricum. J. Exp. Bot. 69, 1955-1966 (2018).

50. Shelton, D. et al. Transcription factors of lotus: regulation of isoflavonoid biosynthesis requires coordinated changes in transcription factor activity. Plant Physiol. 159, 531-547 (2012).

51. Geyter, N. D., Gholami, A., Goormachtig, S. \& Goossens, A. Transcriptional machineries in jasmonate-elicited plant secondary metabolism. Trends Plant Sci. 17, 349-359 (2012).

52. Guo, H. et al. Metabolome and transcriptome association analysis reveals dynamic regulation of purine metabolism and flavonoid synthesis in transdifferentiation during somatic embryogenesis in cotton. Int. J. Mol. Sci. 20, 2070 (2019).

53. Sun, B. et al. Stilbenes: quantitative extraction from grape skins, contribution of grape solids to wine and variation during wine maturation. Anal. Chim. Acta 563, 382-390 (2006).

54. Lin, T. T., Yang, W. N., Lu, W., Wang, Y. \& Qi, X. T. Transcription factors PvERF15 and PVMTF-1 from a cadmium stress transcriptional pathway. Plant Physiol. 173, 1565-1573 (2017).

55. Yang, Y., Li, R. \& Qi, M. In vivo analysis of plant promoters and transcription factors by agroinfiltration of tobacco leaves. Plant J. 22, 543-551 (2000).

56. Jefferson, R. A., Kavanagh, T. A. \& Bevan, M. W. GUS fusions: beta-glucuronidase as a sensitive and versatile gene fusion marker in higher plants. EMBO J. 6 3901-3907 (1987) 